\title{
Late Diagnosis of Anaphylactic Reaction to Gadolinium-Based Contrast Media by Skin Tests 10 Years After Onset
}

Harr T, Jandus P

Division of Immunology and Allergology, Department of Medical Specialties, University Hospital and Medical Faculty, Geneva, Switzerland

J Investig Allergol Clin Immunol 2018; Vol. 28(3): 203-205 doi: $10.18176 /$ jiaci.0240

Key words: Gadolinium-based contrast agents. Magnetic resonance imaging. Skin prick tests. Intradermal tests.

Palabras clave: Gadolinio. Resonancia magnética. Pruebas cutáneas prick. Pruebas intracutáneas.

Gadolinium-based contrast agents (GBCAs) have paramagnetic properties that are used to enhance the diagnostic value of MRI studies. Iodinated contrast media were introduced in the 1920s; however, gadolinium-based contrast agents were first approved for use by the United States Food and Drug Administration (FDA) in 1988 [1].

A 36-year-old white woman presented with sudden onset of dyspnea and reduced consciousness shortly after the application of paramagnetic contrast media (product unknown) during a cerebral MRI in summer 2007. Her condition improved rapidly after treatment with antihistamines, corticosteroids, and intravenous hydration. She has not undergone MRI since then. The patient had been diagnosed with ulcerative colitis and nonallergic asthma. In the following years, she developed Behçet disease and bronchiectasis. She changed physicians several times, with loss of her documentation. In summer 2017, an allergy work-up was performed without documentation to assess the reaction she experienced in 2007. At that time, her Behçet disease and ulcerative colitis were in remission under treatment with colchicine and azathioprine, and her asthma was controlled with budesonide and formoterol.

Ten years after reacting to paramagnetic contrast media, the patient was found to have positive results in skin prick tests (SPT, 1:1) (Figure) and intradermal tests (IDT, 1:100) with gadobutrol (Gadovist) and gadoterate meglumine (Artirem, Dotarem) and negative intradermal test results (IDT, 1:10) with gadobenate dimeglumine (Multihance) and gadopentetate dimeglumine (Magnograf).

SPTs (1:1) and IDTs (1:10) for iodinated contrast media (ioxithalamate, ioxaglate, iohexol, iomeprol, iobitridol, iopromide, iodixanol) were all negative. The basal tryptase level was in the normal range $(3.4 \mu \mathrm{g} / \mathrm{L}, \mathrm{N}<11$; Thermo Fisher). There were no other signs of cutaneous or systemic mastocytosis. Prick tests for atopy were negative with inhalant allergens. Based on the skin test results, hypersensitivity to macrocyclic GBCAs was suspected.

GBCAs have been used in more than 100 million patients worldwide [1]. Immediate hypersensitivity reactions to GBCAs 


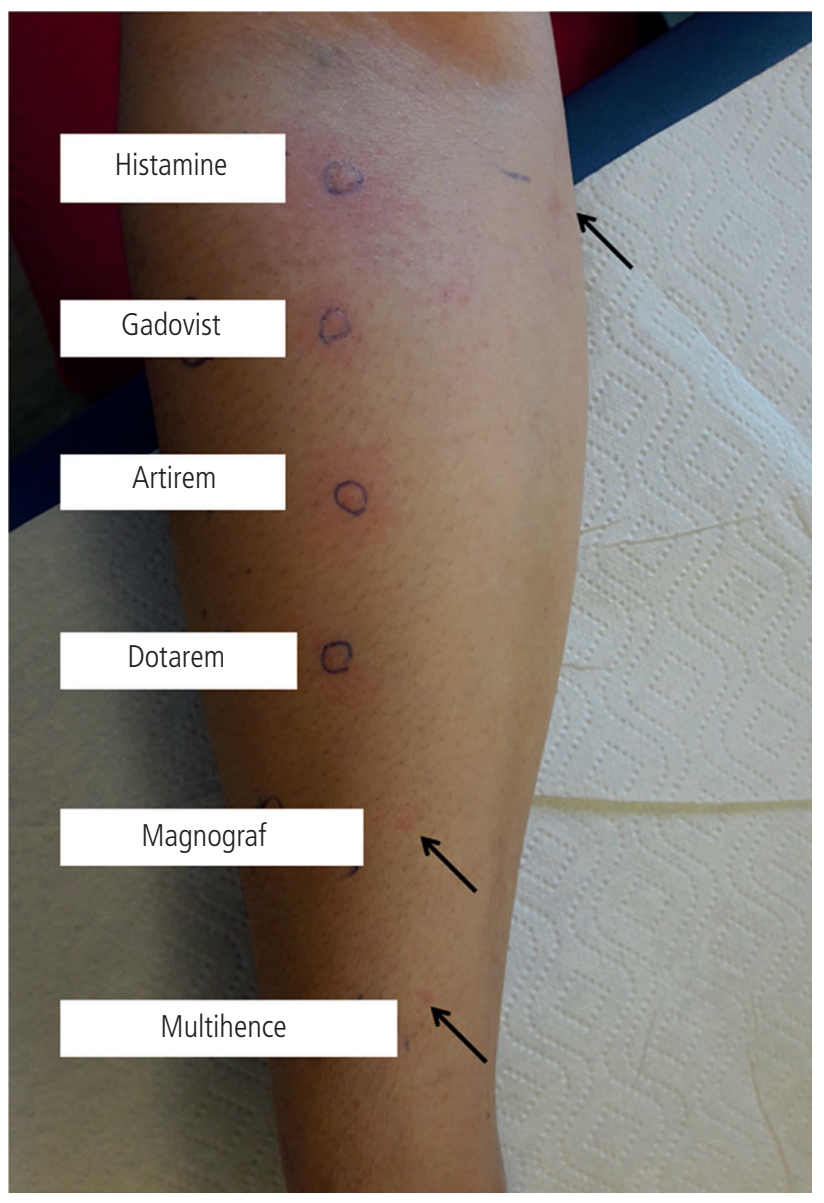

Figure. Positive prick test results with gadolinium-based contrast agents. Arrows indicate areas of skin testing with a negative control and negative skin testing test results for Multihance and Magnograf.

have an estimated adverse event rate of $0.07 \%$ to $2.4 \%$ [2]. Urticaria is the most common manifestation, occurring in up to $90 \%$ of hypersensitivity reactions [3]. With a frequency of $0.004 \%-0.01 \%$ of cases, GBCA-induced anaphylaxis is rare [3]. Patients who have experienced prior immediate reactions to GBCAs have the highest risk for a recurrent reaction upon subsequent readministration, with an estimated frequency of reaction of $30 \%$ [3]. Reactions have been reported to be more frequent after administration of gadobenate dimeglumine and gadoteridol and after abdominal scans $(0.01 \%)$ than brain scans $(0.005 \%)$ or spine scans $(0.003 \%)$ [4]. Patients are at a higher risk of experiencing adverse reactions if they have concomitant atopic diseases, multiple allergies, and prior anaphylactic reactions, as well as after multiple exposures to GBCAs [5]. However, anaphylaxis may occur upon first exposure with no known risk factors, as shown in a case report by Hasdenteufel et al [6]. A mortality rate of $0.0019 \%$ (3 of 158796 cases) has been estimated [5]. The FDA reported an overall mortality rate of $0.00008 \%$ from 2004 to 2009 (40 deaths per 51 million MRI contrast medium doses administered) [5].

In a recent prospective study, 151 patients at risk for reactions were evaluated by skin testing before administration of GBCAs. No positive skin test results were detected, and only
1 patient developed mild urticaria upon administration [7]. Thus, screening of individuals with risk factors does not seem to be effective.

The pathophysiological mechanism of immediate hypersensitivity reactions is not always clear. However, an IgE-mediated mechanism has been suggested based on positive skin test results in patients with immediate reactions to GBCAs [2]. GBCAs have been implicated in the development of nephrogenic systemic fibrosis, particularly in patients with kidney disease. Nonimmediate hypersensitivity reactions to GBCAs are rare and should also be considered [8]. Hypersensitivity reactions to GBCAs should be investigated using SPTs with undiluted GBCAs followed by IDTs at dilutions of 1:1000-1:10 [4]

In patients who have experienced a reaction to GBCAs in the past, recommendations regarding subsequent use of paramagnetic contrast agents are often needed. However, premedication with antihistamines and corticosteroids was not systematically studied in larger patient groups. Furthermore, premedication with antihistamines and corticosteroids was not effective in a small cohort of patients with documented breakthrough reactions [9]. The decision to administer an alternative agent might be facilitated by skin testing. If the responsible agent shows a positive result and a skin testnegative alternative can be found, repeated reaction to the skin test-negative GBCA is unlikely to occur [9]. In this context, the classification of GBCAs into linear and macrocyclic agents based on their molecular structure might facilitate choice [4]. However, alternatives should be assessed with challenge tests to verify tolerance. In studies of patients with immediate hypersensitivity to drugs, skin tests are more likely to be positive the shorter the time elapsed since the clinical reaction. In addition, skin tests are more likely to turn negative the longer the time after the reaction (67.8\% in a 5-year follow-up in cephalosporin hypersensitivity [11]).

Diagnostic criteria are mostly empiric, and consensus guidelines are primarily based on limited case series, observational studies, and expert opinions. GBCAs have received little attention in randomized controlled trials [7]. Here, we report the first case with positive skin test results 10 years after an anaphylactic reaction to GBCAs in an immunosuppressed patient. This case illustrates that hypersensitivity with positive IDT and SPT results might persist over several years. We stress that an undiluted positive SPT result should be considered a true positive. IDT should only be carried out by experienced personnel after negative prick testing, since it entails a risk for the patient and is not necessary after positive SPT results.

A classification of GBCAs into linear and macrocyclic structures might facilitate identification of cross-reactivity. This approach needs to be validated in larger studies.

\section{Funding}

The authors declare that no funding was received for the present study.

\section{Conflicts of Interest}

The authors declare that they have no conflicts of interest. 


\section{References}

1. Fok JS, Smith WB. Hypersensitivity reactions to gadoliniumbased contrast agents. Curr Opin Allergy Clin Immunol. 2017;17:241-6.

2. Carr T. Pathophysiology of Immediate Reactions to Injectable Gadolinium-based Contrast Agents Top Magn Reson Imaging. 2016;25:265-8.

3. Jung JW, Kang HR, Kim MH, Lee W, Min KU, Han MH, Cho SH. Immediate hypersensitivity reaction to gadolinium-based MR contrast media. Radiology. 2012;264:414-22.

4. Rosado Ingelmo A, Doña Diaz I, Cabañas Moreno R, Moya Quesada MC, García-Avilés C, García Nuñez I, et al. Clinical Practice Guidelines for Diagnosis and Management of Hypersensitivity Reactions to Contrast Media. J Investig Allergol Clin Immunol. 2016;26:144-55

5. Prince MR, Zhang H, Zou Z, Staron RB, Brill PW. Incidence of immediate gadolinium contrast media reactions. AJR Am J Roentgenol. 2011;196:W138-43.

6. Hasdenteufel F, Luyasu S, Renaudin JM, Paquay JL, Carbutti $G$, Beaudouin E, et al. Anaphylactic shock after first exposure to gadoterate meglumine: two case reports documented by positive allergy assessment. J Allergy Clin Immunol. 2008;121:527-8.

7. Tepetam FM, Ciftaslan N, Oruc O, Duman D, Agca M, Bulut I, et al. Should patients with risk factors be tested for hypersensitivity to contrast media: a prospective study. Radiol Med. 2016;121:660-7.

8. Nagai $H$, Nishigori C. A delayed reaction to the magnetic resonance imaging contrast agent gadobutrol. J Allergy Clin Immunol Pract. 2017;5:850-1.

9. Dillman JR, Ellis JH, Cohan RH, Strouse PJ, Jan SC. Allergic-like breakthrough reactions to gadolinium contrast agents after corticosteroid and antihistamine premedication. AJR Am J Roentgenol. 2008;190:187.

10. L Chiriac AM, Audurier Y, Bousquet PJ, Demoly P. Clinical value of negative skin tests to gadolinium contrast agents. Allergy. 2011;66:1504-6.

11. Romano A, Gaeta F, Valluzzi RL, Zaffiro A, Caruso C, Quaratino D. Natural evolution of skin-test sensitivity in patients with IgE-mediated hypersensitivity to cephalosporins. Allergy. 2014;69:806-9

I Manuscript received August 13, 2017; accepted for publication February 13, 2018.

Peter Jandus

Division of Immunology and Allergology Department of Medical Specialties University Hospital and Medical Faculty of Geneva Rue Gabrielle Perret-Gentil 4

1211 Geneva 14 Switzerland

E-mail: peter.jandus@hcuge.ch 